\title{
Bubble-mediated transfer of dilute gas in turbulence
}

\author{
Palas Kumar Farsoiya ${ }^{1}$, Stéphane Popinet ${ }^{2}$ and Luc Deike ${ }^{1},{ }^{3} \dagger$, \\ ${ }^{1}$ Department of Mechanical and Aerospace Engineering, Princeton University, Princeton, New \\ Jersey 08544, USA, \\ ${ }^{2}$ Institut Jean Le Rond d'Alembert, CNRS UMR 7190, Sorbonne Université, Paris 75005, \\ France, \\ ${ }^{3}$ High Meadows Environmental Institute, Princeton University, Princeton, NJ 08544, USA
}

(Received xx; revised xx; accepted xx)

Bubble-mediated gas exchange in turbulent flow is critical in bubble column chemical reactors as well as for ocean-atmosphere gas exchange related to air entrained by breaking waves. Understanding the transfer rate from a single bubble in turbulence at large Péclet numbers (defined as the ratio between the rate of advection and diffusion of gas) regimes is important as it can be used for improving the models on a larger scale. We characterize the mass transfer of dilute gases from a single bubble in a homogeneous isotropic turbulent flow in the limit of negligible bubble volume variations. We show that the mass transfer occurs within a thin diffusive boundary layer at the bubble-liquid interface, whose thickness decreases with an increase in turbulent Péclet number, $\widetilde{\text { Pe. We }}$ propose a suitable time scale $\theta$ for Higbie (1935) penetration theory, $\theta=d_{0} / \tilde{u}$, based on $d_{0}$ the bubble diameter and $\tilde{u}$ a characteristic turbulent velocity, here $\tilde{u}=\sqrt{3} u_{\mathrm{rms}}$, where $u_{\mathrm{rms}}$ is the large-scale turbulence fluctuations. This leads to a non-dimensional transfer rate $\mathrm{Sh}=2(3)^{1 / 4} \sqrt{\widetilde{\mathrm{Pe}} / \pi}$, from the bubble in the isotropic turbulent flow. The theoretical prediction is verified by direct numerical simulations of mass transfer of dilute gas from a bubble in homogeneous and isotropic turbulence, and very good agreement is observed as long as the thin boundary layer is properly resolved.

\section{Introduction}

The evolution of bubbles in turbulence has multiple applications in environmental and industrial contexts, from exchange at the ocean-atmosphere interface (Deike et al. 2016; Deike \& Melville 2018), as bubble-mediated gas exchange accounts for a significant part of carbon dioxide $\left(\mathrm{CO}_{2}\right)$ uptake by the ocean (Reichl \& Deike 2020), to bubble column chemical reactors (Risso 2018), while providing an effective pathway to bring oxygen in lakes (Karn et al. 2015). In an engineering context, bubble-mediated gas exchange is controlled by the level of agitation in the flow, which is itself influenced by the presence of bubbles, and in turn affects the bubble velocity statistics controlling the gas transfer from individual bubbles (Risso 2018; Mathai et al. 2020).

While empirical formulae (e.g. Karn et al. (2015); Colombet et al. (2015)) have been proposed to describe the gas transfer in bubble swarms, their general applicability and theoretical foundation remain an active research topic. Bubble-mediated gas transfer models in the context of ocean-atmosphere interaction often use formulae based on gas transfer theory (Levich 1962) based on bubble rising in quiescent flow (Woolf \& Thorpe 1991; Keeling 1993; Liang et al. 2011; Deike \& Melville 2018). The diffusive gas transfer by

$\dagger$ Email address for correspondence: ldeike@princeton.edu 
a single bubble rising in a quiescent flow has been described theoretically by Boussinesq (1905) and Levich (1962), within the assumption of negligible variation in bubble volume. The non-dimensional transfer rate from a single bubble is the Sherwood number Sh= $k_{L} d_{0} / \mathscr{D}_{l}$, where $k_{L}$ is the transfer rate, $d_{0}$ the bubble size and $\mathscr{D}_{l}$ the gas diffusivity in the liquid, and reads, $\mathrm{Sh}=\frac{2}{\sqrt{\pi}} \sqrt{\mathrm{Pe}}$ where $\mathrm{Pe}=U d_{0} / \mathscr{D}_{l}$ is the bubble Péclet number and $U$ the bubble rise velocity. Several studies dealing with mass transfer from bubbles rising in a quiescent liquid have developed numerical techniques to resolve interphase mass transfer and validated their results against diffusive mass transfer theory (Haroun et al. 2010; Marschall et al. 2012; Deising et al. 2016; Fleckenstein \& Bothe 2015; Deising et al. 2018). Direct numerical simulations (DNS) of mass transfer of dilute gas from rising bubble swarms by Roghair (2012) concluded that the transfer rate increases as the gas hold-up (ratio of gas volume to the total volume) increases.

The gas transfer at the interface between two fluids involving a turbulent flow has been described as presenting two regimes depending on the turbulent Reynolds number (Theofanous et al. 1976). At low-Reynolds number, the transfer rate can be written as $k_{L} / u_{0} \propto \mathrm{Sc}^{-1 / 2} \mathrm{Re}^{-1 / 2}$, where $\mathrm{Sc}=\nu / \mathscr{D}_{l}$ is the Schmidt number (ratio of kinematic viscosity over mass diffusivity), $u_{0}$ a characteristic turbulent velocity and Re a turbulence Reynolds number. The high-Reynolds number regime can be described through the action of the smallest eddies at the Kolmogorov scale enhancing the transfer, leading to $k_{L} \sim \mathrm{Sc}^{-1 / 2}(\nu \epsilon)^{1 / 4}$, where $\epsilon$ is the turbulence dissipation rate. This can be expressed in terms of the turbulence Reynolds number and reads $k_{L} / u_{0} \propto \mathrm{Sc}^{-1 / 2} \mathrm{Re}^{-1 / 4}$ (Theofanous et al. 1976), further discussed by Magnaudet \& Calmet (2006); Katul \& Liu (2017). These two regimes and their crossover have been observed experimentally and numerically by Herlina \& Wissink $(2016,2019)$, following earlier experimental work by Fortescue \& Pearson (1967) (who used the root mean square of the fluctuating velocity as characteristic velocity).

The same reasoning can be applied in the context of bubbles in a turbulent flow. Levich (1962) provides a brief discussion on gas dissolution from a bubble of diameter $d_{0}$ in a turbulent stream with a characteristic velocity $u_{0}$. The gas transfer rate can then be estimated as $\mathrm{Sh} \propto \mathrm{Re}^{3 / 4} \mathrm{Sc}^{1 / 2}, \mathrm{Sc}=\nu / \mathscr{D}_{l}$ is the Schmidt number (ratio of kinematic viscosity over mass diffusivity) and $\operatorname{Re}=d_{0} u_{0} / \nu$ a turbulence Reynolds number, based on the bubble size $d_{0}$ and a turbulence velocity $u_{0}$ (the velocity scale considered by Levich (1962) is the maximum velocity of the eddies in the liquid that flows past the bubble). This regime is equivalent in terms of scalings to the high Reynolds number regime described by Theofanous et al. (1976); Magnaudet \& Calmet (2006) and its applicability in the context of mass exchange by a bubble swarm is discussed by Colombet et al. (2015).

Numerical methods for interfacial mass transfer started with (Sato et al. 2000; Davidson \& Rudman 2002) where the dilute gas concentration is continuous across the interface. Bothe et al. (2004) introduced a method to simulate the discontinuous concentration due to solubility. A three-dimensional front tracking model with mass transfer was presented by Darmana et al. (2006). A one-fluid formulation for the algebraic volume of fluid method was presented independently by Haroun et al. (2010) and Marschall et al. (2012). Bothe \& Fleckenstein (2013) introduced a two-field approach using a geometrical volume of fluid method for multicomponent conjugate mass transfer. Sub-grid scale models to simulate high-Schmidt-number, bubble-mediated mass exchange have been developed by Bothe \& Fleckenstein (2013); Weiner \& Bothe (2017); Claassen et al. (2020). Recent advances in numerical methods by Tanguy et al. (2014); Fleckenstein \& Bothe (2015); Maes \& 
Soulaine (2020); Scapin et al. (2020) allow to simulate problems of mass transfer with local volume changes.

In the present work, we adapt the classic penetration theory (Higbie 1935) to describe the mass transfer from a bubble in a turbulent flow, by considering a turbulent time scale and predict the transfer rate of low-solubility gases, presented in $\S 2$. We next present a framework in $\S 3$ which combines recent advances in numerical algorithms for interfacial mass transfer, together with progress in turbulent multiphase flow modeling to characterize the diffusive mass transfer of dilute gas from a bubble in turbulence. Dilute gas diffusion from a bubble is similar to conjugate mass transfer from a spherical droplet (Rachih et al. 2020). However, in the present study, the fluid inside the spherical cavity is considerably less dense than the surrounding fluid and hence is called a bubble. We use the Basilisk flow solver (Popinet \& collaborators 2013-2020) which uses adaptive mesh refinement, a momentum-conserving scheme for velocity and a geometric volume-of-fluid method to capture the interface. We implement a module for the concentration advection and diffusion using one-fluid formulation without phase change (Haroun et al. 2010; Bothe \& Fleckenstein 2013; Taqieddin 2018; Yang et al. 2020). The mass transfer module is validated by comparing the numerical results of diffusion from a static bubble with a solution using inverse Laplace transform, as well as for the classic diffusion from a rising bubble in a quiescent liquid. Finally, in $\S 4$, we perform DNS of bubble-mediated mass transfer in a homogeneous isotropic turbulent (HIT) flow, solving the three-dimensional, incompressible, two-phase Navier-Stokes equations coupled with an advection-diffusion equation for the gas concentration. We consider bubbles that can deform but at Weber number below the break-up threshold. The theoretical model is confirmed by the DNS results for a wide range of turbulent Péclet numbers.

The present configuration of mass exchange of a dilute component from a bubble to the surrounding turbulent water is especially relevant for bubble mediated $\mathrm{CO}_{2}$ gas transfer at the ocean-atmosphere interface, as $\mathrm{CO}_{2}$ is present in a small concentration in the atmosphere so that its exchange with the surrounding water does not change the overall bubble volume, while gases such as $\mathrm{N}_{2}$ and $\mathrm{O}_{2}$ which contribute for most of the volume of the bubbles have a much lower solubility and exchange over longer times (see detailed discussions in Woolf \& Thorpe (1991); Keeling (1993); Liang et al. (2011); Deike \& Melville (2018)).

\section{Theory of bubble mass transfer in turbulence}

We consider the mass transfer of dilute gas from a bubble of diameter $d_{0}$, the diffusivity of gas inside and outside the bubble is given by $\mathscr{D}_{g}$ and $\mathscr{D}_{l}$ respectively. The ratio of momentum to mass diffusivity defines the Schmidt number $\mathrm{Sc}=\nu_{l} / \mathscr{D}_{l}$. The mass transfer predicted by the two-film theory (Whitman 1923) assumes a steady-state diffusion through the film, while in turbulent flows the fluctuations in the velocity field keep the diffusion transient (Treybal 1980). Higbie (1935) penetration theory states that when eddies of liquid in a turbulent flow are exposed to bubbles for a timescale $\theta$, then the mass transfer rate is given by,

$$
k_{L}=\frac{2}{\sqrt{\pi}} \sqrt{\frac{\mathscr{D}_{l}}{\theta}} .
$$

In a turbulent flow, the eddies of various sizes will interact with the bubble and advect the gas present in the surrounding thin boundary layers. These interactions will be characterized by a turbulent velocity $\tilde{u}$. Several choices could be considered, in particular 
the large scale fluctuation velocity, the velocity fluctuations at the bubble scale or the small scale fluctuations. We consider here that the large scale fluctuations of the flow will drive the transfer process, and can be characterized by the magnitude of velocity fluctuations $|\tilde{u}|=\sqrt{3} u_{\mathrm{rms}}$. We propose that the time scale of exposure to eddies is given by $\theta=d_{0} /\left(\sqrt{3} u_{\mathrm{rms}}\right)$. The transfer rate, Eq. 2.1, is then,

$$
k_{L}=\frac{2(3)^{1 / 4}}{\sqrt{\pi}} \sqrt{\frac{\mathscr{D}_{l} u_{\mathrm{rms}}}{d_{0}}}
$$

For a bubble-mediated mass transfer in turbulent flow, we define the non-dimensional transfer rate, or Sherwood number, Sh $=k_{L} d_{0} / \mathscr{D}_{l}$, and the turbulent Péclet number, $\widetilde{\mathrm{Pe}}=u_{\mathrm{rms}} d_{0} / \mathscr{D}_{l}$. Finally, Eq. 2.2, can be written,

$$
\mathrm{Sh}=\frac{2(3)^{1 / 4}}{\sqrt{\pi}} \sqrt{\widetilde{\mathrm{Pe}}}
$$

This equation is analogous to that for the transfer rate for a bubble rising in a quiescent fluid, where the Péclet number would be defined based on the bubble rise velocity (Levich 1962) and mass transfer for bubble swarms where the mean rise velocity of the bubbles in the swarm is considered (Colombet et al. 2015).

\section{Numerical Framework}

\subsection{The Basilisk solver}

We solve the three-dimensional, incompressible, two-phase Navier-Stokes equations using the open-source solver Basilisk (Popinet 2009, 2015; van Hooft et al. 2018),

$$
\begin{aligned}
& \partial_{t} \mathbf{u}+\nabla \cdot(\mathbf{u u})=\frac{1}{\rho}\left[-\nabla p+\nabla \cdot\left(\mu\left(\nabla \mathbf{u}+\nabla \mathbf{u}^{T}\right)\right)\right]+\frac{\gamma}{\rho} \kappa \delta_{s} \mathbf{n}, \\
& \nabla \cdot \mathbf{u}=0 \\
& \frac{\partial \mathcal{T}}{\partial t}+\mathbf{u} \cdot \nabla \mathcal{T}=0 .
\end{aligned}
$$

where $\mathbf{u}, p, \gamma, \mu, \rho, \kappa, n$ and $\mathcal{T}$ are the velocity, pressure, surface tension coefficient, viscosity, density, curvature, interface normal and volume fraction fields respectively. The solver has been extensively validated for complex interfacial flows (Popinet 2015; Farsoiya et al. 2017; Gumulya et al. 2020; Ruth et al. 2019; Mostert \& Deike 2020; Berny et al. 2020). It uses the projection method to compute the velocity and pressure and the geometric volume of fluid ( VoF) method for the evolution of the interface between two immiscible fluids (Tryggvason et al. 2011). The Piecewise Linear Interface Calculation (PLIC) geometric interface and flux reconstruction ensures a sharp representation of the interface (Scardovelli \& Zaleski 1999; Maric et al. 2020) and is combined with an accurate Height-Function curvature calculation and a well-balanced, continuum surface tension model (Brackbill et al. 1992; Popinet 2018).

\subsection{One fluid formulation for mass transfer of dilute gas}

The continuous formulation for mass transfer which we use in this study has been independently developed by Haroun et al. (2010) and Marschall et al. (2012). We have implemented the concentration diffusion of dilute gas (Haroun et al. 2010) using the harmonic mean diffusion coefficient as verified by Deising et al. (2016). Note that the 
present study investigates the mass transfer of dilute gas present in the bubble where the effect of loss of volume and phase change are ignored. Numerical methods for phase change require changes in the volume of fluid advection equation and continuity equation (Tanguy et al. 2014; Fleckenstein \& Bothe 2015; Maes \& Soulaine 2020; Scapin et al. 2020). The time evolution of the $j$ th gas concentration, $c_{l / g, j}$, for the liquid phase $l$ or the gas phase $g$ is given by (Standart 1964; Haroun et al. 2010)

$$
\frac{\partial c_{l / g, j}}{\partial t}+\nabla \cdot\left(\mathbf{u} c_{l / g, j}\right)=-\nabla \cdot\left(J_{l / g, j}\right) .
$$

The continuity of normal fluxes across the interface $\Sigma$ (Standart 1964; Bothe \& Fleckenstein 2013),

$$
\llbracket\left(c_{j}\left(\mathbf{u}-\mathbf{u}_{\Sigma}\right)+\mathbf{J}_{j}\right) \cdot \mathbf{n}_{\Sigma} \rrbracket=0
$$

where $\mathbf{u}_{\Sigma}$ is the interface velocity. As we assume that the transfer of a dilute component does not cause volume change, (3.5) reduces to,

$$
\mathbf{J}_{l, j} \cdot \mathbf{n}_{\Sigma}=\mathbf{J}_{g, j} \cdot \mathbf{n}_{\Sigma}
$$

The standard assumption of continuous chemical potentials which is good for most applications at interface $\Sigma$ results in Henry's law, (we refer interested readers to Bothe \& Fleckenstein (2013) for the discussion on local chemical equilibrium and the generalized Henry's law),

$$
c_{l, j}=c_{g, j} \alpha_{j}
$$

where the dimensionless ratio of the liquid phase concentration to the gas phase concentration of the component transferred $\alpha_{j}$, is called Henry's law solubility constant (Sander 2015) (solubility hereafter). The problem investigated is isothermal and there is no bubble breakup or large deformation of the interface which can change the pressure inside the bubble significantly. Hence, solubility which at least depends on temperature and pressure (Bothe \& Fleckenstein 2013), is assumed to be constant. Introducing variables for the one-fluid formulation which is valid for both phases $l$ and $g$ and for gas $j$, we get,

$$
c_{j}=\mathcal{T} c_{l}+(1-\mathcal{T}) c_{g}, \quad J_{j}=\mathcal{T} J_{l}+(1-\mathcal{T}) J_{g}
$$

and the flux is given by

$$
J_{j}=-\left(\mathcal{T} \mathscr{D}_{j l} \nabla c_{l}+(1-\mathcal{T}) \mathscr{D}_{j g} \nabla c_{g}\right) .
$$

The diffusivity for the interfacial cells is calculated using the harmonic mean of the two diffusivities of the gas inside and outside the bubble (Haroun et al. 2010),

$$
\mathscr{D}_{j}=\frac{\mathscr{D}_{j g} \mathscr{D}_{j l}}{\mathscr{D}_{j l}(1-\mathcal{T})+\mathscr{D}_{j g} \mathcal{T}},
$$

to get a single equation for both phases,

$$
\frac{\partial c_{j}}{\partial t}+\nabla \cdot\left(\mathbf{u} c_{j}\right)=\nabla \cdot\left(\mathscr{D}_{j} \nabla c_{j}-\mathscr{D}_{j}\left(\frac{c_{j}\left(\alpha_{j}-1\right)}{\alpha_{j} \mathcal{T}+(1-\mathcal{T})}\right) \nabla \mathcal{T}\right)
$$

The coefficient of $c_{j}$ in the second term containing the right hand side of Eq. (3.11) can be written as $\beta_{j}$,

$$
\frac{\partial c_{j}}{\partial t}=\nabla \cdot\left(\mathscr{D}_{j} \nabla c_{j}+\beta_{j} c_{j}\right)
$$


Using a time-implicit Euler discretisation,

$$
\frac{c_{j}^{n+1}-c_{j}^{n}}{\Delta t}=\nabla \cdot\left(\mathscr{D}_{j} \nabla c_{j}^{n+1}+\beta_{j} c_{j}^{n+1}\right)
$$

and rearranging the implicit terms, gives

$$
\nabla \cdot\left(\mathscr{D}_{j} \nabla c_{j}^{n+1}+\beta_{j} c_{j}^{n+1}\right)-\frac{c_{j}^{n+1}}{\Delta t}=-\frac{c_{j}^{n}}{\Delta t},
$$

Eq. (3.14) is a set of linear equations which is solved efficiently using the multigrid method (Popinet 2015).

The solubility boundary condition (3.7) presents a discontinuity for the concentration field across the interface similar to the volume fraction field $\mathcal{T}$. As discussed by Bothe \& Fleckenstein (2013), a non-consistent advection leads to artificial diffusion for concentration. For a consistent advection two tracer fields $\phi_{g}$ and $\phi_{l}$ associated with the volume of fluid $\mathcal{T}$ are defined,

$$
\phi_{l}=c_{l} \mathcal{T}, \quad \phi_{g}=c_{g}(1-\mathcal{T})
$$

Using Eq. (3.7) and Eq. (3.8), we get,

$$
\phi_{l, j}=\frac{c_{j}^{n+1} \alpha_{j} \mathcal{T}}{\alpha_{j} \mathcal{T}+(1-\mathcal{T})}, \quad \phi_{g, j}=\frac{c_{i}^{n+1}(1-\mathcal{T})}{\alpha_{j} \mathcal{T}+(1-\mathcal{T})}
$$

The advection equation for $\phi_{g / l}$ reads,

$$
\frac{\partial \phi_{g / l}}{\partial t}+\nabla \cdot\left(\mathbf{u} \phi_{g / l}\right)=0
$$

and is solved using the volume-of-fluid associated fields (López-Herrera et al. 2015) which guarantees strictly non-diffusive transport close to the interface. The concentration tracer is updated after advection using

$$
c_{j}=\phi_{g, j}+\phi_{l, j}
$$

\subsection{Validation}

We validate the numerical methods and their implementation for static and rising bubble cases.

\subsubsection{Diffusion from a static bubble}

We propose a new test case of diffusion from a constant-size, static, spherical bubble. Test cases are available for concentration profiles in the case of a planar interface in Bird et al. (2002); Haroun et al. (2010). We provide the solution for the transient concentration both inside and outside the spherical bubble in the form of integrals which are then evaluated numerically.

Consider a static axisymmetric bubble of radius $R_{0}=d_{0} / 2$, the diffusivities of the gas are $D_{g}$ and $D_{l}$ for the gas and liquid phase respectively. The one-dimensional transient concentration diffusion for a spherical geometry for both inside and outside the bubble is given by

$$
\frac{\partial c_{g}}{\partial t}=\mathscr{D}_{g} \frac{1}{r^{2}} \frac{\partial}{\partial r}\left(r^{2} \frac{\partial c_{g}}{\partial r}\right), \quad \frac{\partial c_{l}}{\partial t}=\mathscr{D}_{l} \frac{1}{r^{2}} \frac{\partial}{\partial r}\left(r^{2} \frac{\partial c_{l}}{\partial r}\right) .
$$

Applying the Laplace transform to the above equations, we get, $\tilde{c}(s)=\mathcal{L}[c(t)]$,

$$
\frac{d^{2} \tilde{c}_{g}}{d r^{2}}+\frac{2}{r} \frac{d \tilde{c}_{g}}{d r}-\frac{s}{\mathscr{D}_{g}} \tilde{c}_{g}+\frac{1}{\mathscr{D}} c_{g 0}=0, \quad \frac{d^{2} \tilde{c}_{l}}{d r^{2}}+\frac{2}{r} \frac{d \tilde{c}_{l}}{d r}-\frac{s}{\mathscr{D}_{l}} \tilde{c}_{l}+\frac{1}{\mathscr{D}} c_{l 0}=0,
$$



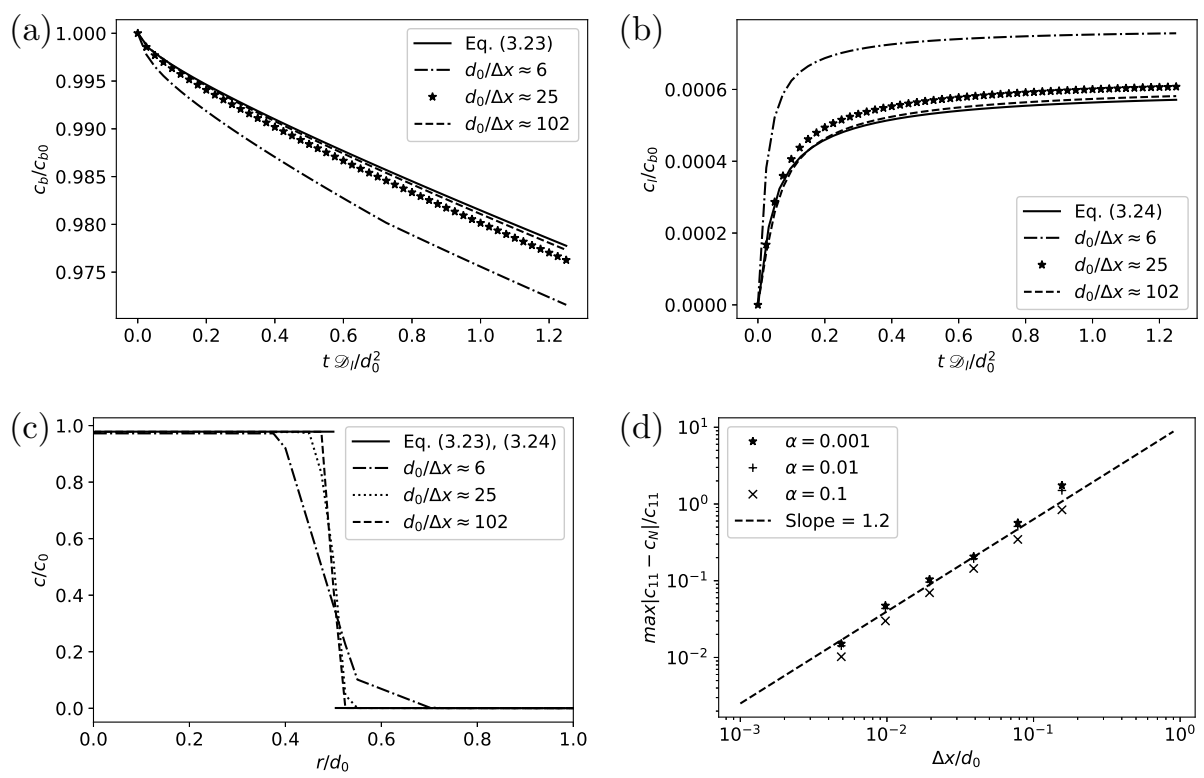

Figure 1: Diffusion from a static bubble, comparing the numerical results with (3.23) and (3.24). (a) Concentration inside the bubble at $r / d_{0}=0.25$. (b) Concentration outside the bubble at $r / d_{0}=0.75$. (c) Radial profile at time $t \mathscr{D}_{l} / d_{0}^{2}=1.2$. (d) Maximum relative error at different resolutions $\max \left|c_{11}-c_{n}\right| / c_{11}$, where $c_{11}$ and $c_{n}$ are numerical solutions at resolution $2^{11}$ and lower respectively, and for different solubilities $\alpha$, displaying first order convergence. The scripts sufficient to reproduce these results are provided in Farsoiya et al. (2020a).

given boundary conditions, $r \tilde{c}_{g} \rightarrow 0$ as $r \rightarrow 0, \tilde{c}_{l} \rightarrow 0$ as $r \rightarrow \infty, \llbracket \mathscr{D} \partial \tilde{c} / \partial r \rrbracket=0$ and $\tilde{c}_{l} / \tilde{c}_{g}=\alpha$ at $r=R_{0}$, the concentrations (Laplace transformed) inside the bubble $\tilde{c}_{g}$ and outside $\tilde{c}_{l}$ are given by

$$
\tilde{c}_{g}(s, r)=\frac{c_{g 0}}{s}\left(1-\frac{2}{\zeta(s) r} \sinh \left[\lambda_{g}(s) r\right]\right), \quad \tilde{c}_{l}(s, r)=\frac{\xi c_{g 0}}{s \zeta(s) r} \exp \left[-\lambda_{l}(s) r\right]
$$

where,

$$
\begin{aligned}
& \lambda_{g / l}=\sqrt{\frac{s}{\mathscr{D}_{g / l}}}, \quad \zeta(s)=\frac{\xi \exp \left[-\lambda_{l} R_{0}\right]}{\alpha R_{0}}+\frac{2}{R_{0}} \sinh \left(\lambda_{g} R_{0}\right) \\
& \xi(s)=\frac{2 \mathscr{D}_{g}\left(\lambda_{g} R_{0} \cosh \left(\lambda_{g} R_{0}\right)-\sinh \left(\lambda_{g} R_{0}\right)\right)}{\mathscr{D}_{l} \exp \left[-\lambda_{l} R_{0}\right]\left(1+\lambda_{l} R_{0}\right)}, \quad c_{g 0}=c_{g}(0, r), \quad c_{l 0}=0 .
\end{aligned}
$$

Eq. 3.21, can be inverted using Cauchy residue theorem (using keyhole contour as discussed in Farsoiya et al. (2020)),

$$
\begin{aligned}
& c_{g}(t, r)=-\frac{2 c_{g 0}}{\pi r} \int_{0}^{\infty} \frac{1}{x} \operatorname{Im}\left\{\frac{\sinh \left(\lambda_{g}(-x) r\right)}{\zeta(-x)}\right\} \exp [-x t] d x, \\
& c_{l}(t, r)=\frac{c_{g 0}}{\pi r} \int_{0}^{\infty} \frac{1}{x} \operatorname{Im}\left\{\frac{\xi(-x)}{\zeta(-x)} \exp \left[-\lambda_{l}(-x) r\right]\right\} \exp [-x t] d x,
\end{aligned}
$$

where $\operatorname{Im}(\cdot)$ is the imaginary part of a complex number. We validate the results of the 
Basilisk solver against (3.23) and (3.24) (using the numerical integration functions based on double exponential quadrature in Wolfram Research, Inc. (2020)) in Fig. 1.

A static bubble of diameter $d_{0} / L=0.2$, diffusivity ratio $\mathscr{D}_{g} / \mathscr{D}_{l}=10$ and solubility $\alpha=10^{-3}$ to $10^{-1}$ is considered for this test. Figure $1(\mathrm{a})$ and 1 (b) show the transient concentrations inside the bubble at $r / d_{0}=0.25$ and outside the bubble at $r / d_{0}=0.75$ respectively. The accuracy of the numerical solution is very good above 25 cells per diameter. The stringent solubility condition $\alpha=10^{-3}$ is achieved at the interface $r / d_{0}=0.5$ even at a low resolution of six cells per diameter (figure $1(\mathrm{c})$ ). To quantify the convergence, we compute the norm, $\max \left|c_{11}-c_{n}\right| / c_{11}$ at different resolutions where $c_{11}$ and $c_{n}$ are numerical solutions at uniform resolutions $2^{11} \times 2^{11}$ and lower respectively. Figure 1(d) shows first-order of convergence with respect to the grid size $\Delta x$. The effect of solubility on the error has not been discussed in earlier studies. We show in Figure 1(d) that decreasing the solubility at the interface increases the error in the solution for a given resolution, but that the error remain small at high resolution.

\begin{tabular}{|c|c|c|c|c|c|c|c|c|c|c|c|c|}
\hline & \multirow{2}{*}{$\begin{array}{l}\text { Mo } \\
\operatorname{Ar} \\
\approx(100- \\
8000)\end{array}$} & \multirow[t]{2}{*}{ Bo } & \multicolumn{5}{|c|}{$\mathrm{Re}$} & \multicolumn{5}{|c|}{$\mathrm{Sh}$} \\
\hline & & & $\begin{array}{l}\text { Clift } \\
\text { et al. } \\
(2005)\end{array}$ & $\begin{array}{l}\text { Roghair } \\
(2012)\end{array}$ & $\begin{array}{l}\text { Deising } \\
\text { et al. } \\
(2016)\end{array}$ & $\begin{array}{l}\text { PW: } \\
\text { Axi }\end{array}$ & $\begin{array}{l}\text { PW: } \\
3 \mathrm{D}\end{array}$ & $\begin{array}{l}\text { Levich } \\
(1962)\end{array}$ & $\begin{array}{l}\text { Roghair } \\
(2012)\end{array}$ & $\begin{array}{l}\text { Deising } \\
\text { et al. } \\
(2016)\end{array}$ & PW: Axi & PW: 3D \\
\hline $\mathrm{A}$ & $10^{-4}$ & 1.0 & 5 & 5.53 & 6.4 & 5.6 & 5.6 & 2.5 & 3.2 & 3.6 & $3.07-3.19$ & $3.17-3.25$ \\
\hline B & $5 \cdot 10^{-4}$ & 3.125 & 10 & 11.31 & 10.5 & 10.9 & 10.9 & 3.7 & 4.4 & 4.2 & 3.99-4.09 & $4.07-4.09$ \\
\hline $\mathrm{C}$ & $9.2 \cdot 10^{-3}$ & 40 & 33 & 32.35 & 32.5 & 32.8 & 32.9 & 6.4 & 6.3 & 6.4 & $4.63-5.42$ & $5.25-6$ \\
\hline $\mathrm{D}_{1}$ & $5 \cdot 10^{-7}$ & 3.125 & 103 & 102.78 & 97.7 & 102.4 & 102.9 & 11.5 & 12.03 & 11.5 & $10.52-10.98$ & $11.15-11.33$ \\
\hline $\mathrm{D}_{2}$ & $5 \cdot 10^{-7}$ & 3.125 & 103 & 102.78 & 97.7 & 102.4 & 102.9 & 36.5 & - & - & $33.12-37.10$ & $35.07-36.38$ \\
\hline $\mathrm{D}_{3}$ & $5 \cdot 10^{-7}$ & 3.125 & 103 & 102.78 & 97.7 & 102.4 & 102.9 & 114.7 & - & - & $\begin{array}{l}108.10 \\
122.13\end{array}$ & $\begin{array}{l}116.91- \\
121.18\end{array}$ \\
\hline
\end{tabular}

Table 1: Reynolds and Sherwood numbers (for $t U / d_{0} \geq 2$ ) for axisymmetric and 3D rising bubbles obtained from our numerical study, indicated as present work (PW Axi, and PW 3D), compared with existing theoretical (Clift et al. 2005; Levich 1962) and numerical results (Roghair 2012; Deising et al. 2016).

\subsubsection{Diffusion from a rising bubble}

For the validation of the advection-diffusion scheme, we consider a bubble in a quiescent fluid rising due to buoyancy. We set up the test cases with the parameters considered in earlier studies (Darmana et al. 2006; Roghair 2012; Deising et al. 2016; Jia et al. 2019), which encompass significant variations in bubble conditions, through variations of the Bond and Archimedes (or Morton) numbers. The rise velocity of a bubble in a quiescent liquid is indeed determined by the bubble-liquid physical parameters, summarized by the Archimedes number $\mathrm{Ar}=g d_{0}^{3} \rho_{l}\left(\rho_{l}-\rho_{g}\right) / \mu^{2}$ or Morton number Mo $=g \mu_{l}^{4} /\left(\rho_{l} \gamma^{3}\right)$ and Bond number Bo $=\rho_{l} g d_{0}^{2} \gamma$ (Moore 1965; Maxworthy et al. 1996; Clift et al. 2005; CanoLozano et al. 2016). The terminal rise velocity $U$ can be computed theoretically (Moore 1965; Clift et al. 2005), and expressed as a non-dimensional bubble Reynolds number $\operatorname{Re}=\rho_{l} U d_{0} / \mu_{l}$. We consider four bubble configurations leading to Re from 5 to 100 , for Bond numbers ranging from 1 to 40, and Archimedes numbers ranging from 100 to 8000 . The mass transfer for these bubble conditions is then computed, considering the ratio of momentum to mass diffusivity given by the Schmidt number $\mathrm{Sc}=\nu_{l} / \mathscr{D}_{l}=1$ and the gas solubility $\alpha=1 / 30$, as in Deising et al. (2016). The fourth configuration is further tested for high Schmidt numbers of 10 and 100 in $\mathrm{D}_{2}$ and $\mathrm{D}_{3}$ respectively. Our results are shown in figure 2 and validated against previous work as shown in Table 1 . Note that to achieve the correct mass transfer, the correct rise velocity must be obtained, since 

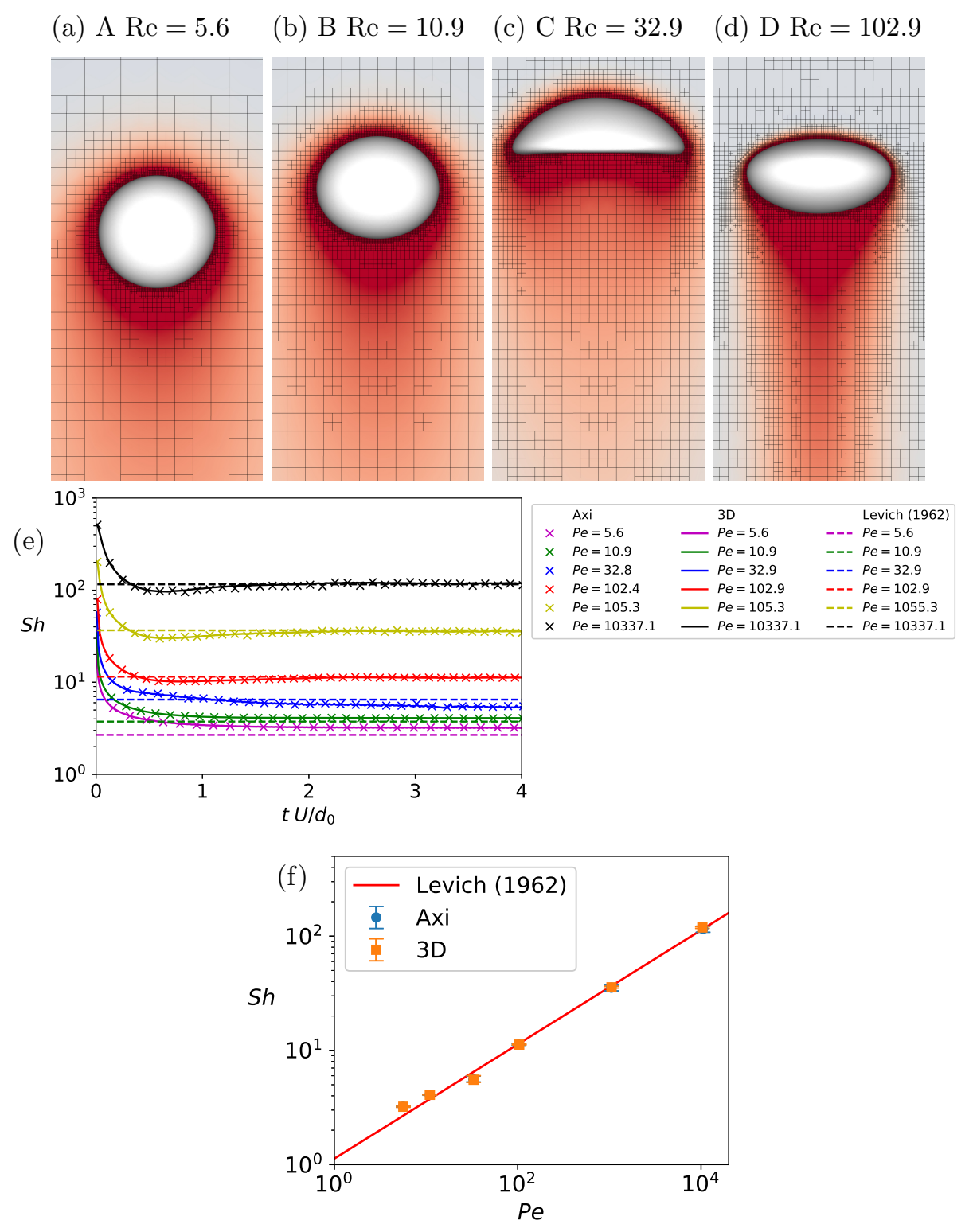

Figure 2: Mass transfer of dilute gas from a bubble rising in a quiescent flow. (a)-(d) Concentration $c$ and bubble interface (three dimensional) at $t U / d_{0} \approx 4$ for the four cases considered (increasing bubble Re number). (e) Evolution with time of transfer rates for axisymmetric and three-dimensional simulations and from Levich (1962) using the computed terminal velocity, (f) Steady state transfer rate (for $t U / d_{0} \geq 2$ ) as a function of Péclet number compared against Levich (1962). Very good agreement between the theoretical and numerical mass transfer is observed except for case $\mathrm{C}$ where the terminal shape is far from spherical. The scripts sufficient to reproduce these results are provided in Farsoiya et al. (2020b). 
the mass transfer will directly depend on the bubble velocity. Figure 2 (a to d) shows examples of the shape of the bubble and the surrounding adaptive grid once the terminal velocity is reached. The average gas concentrations inside and outside the bubble are computed as

$$
\bar{c}_{g, j}=\frac{1}{V_{g}} \int_{V_{g}} c_{j} d V, \quad \bar{c}_{l, j}=\frac{1}{V_{l}} \int_{V_{l}} c_{j} d V
$$

where $V_{g}$ and $V_{l}$ are the volumes of bubble and liquid respectively. The mass transfer rate $k_{L}$ is calculated as,

$$
k_{L}=\frac{\bar{c}_{g, j}^{n+1}-\bar{c}_{g, j}^{n}}{A_{g} \Delta t\left(\alpha \bar{c}_{g, j}^{n+1 / 2}-\bar{c}_{l, j}^{n+1 / 2}\right)}
$$

where $A_{g}$ is the instantaneous surface area of the bubble. The non-dimensionalized transfer rates are computed for both the axisymmetric and three-dimensional simulations with adaptive mesh refinement and a maximum refinement corresponding to a resolution on the bubble of $d_{0} / \Delta x=100$. Levich (1962) derived the mass transfer rates from a rising bubble with constant size and surface concentration, which reads, Sh $=\frac{2}{\sqrt{\pi}} \sqrt{\mathrm{Pe}}$ where $\mathrm{Pe}=\mathrm{Re} \mathrm{Sc}$, is the bubble Péclet number. Upon reaching terminal velocity the transfer rates are steady after $t U / d_{0} \geq 2$ as shown in figure $2(\mathrm{e})$ and are accurately predicted by Levich (1962) as shown in figure $2(\mathrm{e})$ and $2(\mathrm{f})$. The values of the non-dimensional transfer rate, scaled by the diffusion velocity scale, or Sherwood number $\mathrm{Sh}=k d_{0} / \mathscr{D}_{l}$ are also provided in Table 1. Overall, the agreement between theoretical and numerical transfer rates is very good. The deviation from the spherical shape in case $\mathrm{C}$, visible in Figure 2(c), causes a difference with the predicted transfer rate which assumes a spherical bubble shape.

\section{Mass transfer in homogeneous and isotropic turbulence}

We now present DNS of diffusion of dilute gas from a bubble inside a surrounding homogeneous and isotropic turbulent flow (HIT). We follow recent work on bubble deformation in turbulence (Perrard et al. 2021; Rivière et al. 2021) and first prepare the turbulent flow by solving the momentum equation with a forcing term, and then insert a bubble at the center of the HIT flow, once a statistically stationary state has been reached. A similar approach has been used to study the interaction of droplets with isotropic turbulence (Dodd \& Ferrante 2016; Elghobashi 2019a). As the bubble is inserted, the volume of fluid advection Eq. (3.3) and mass advection-diffusion Eq. (3.11) is solved coupled with the momentum equations Eq. (3.1). We then calculate the mass transfer rate from the bubble and compare it with the theory presented in $\S 2$.

\begin{tabular}{cccccccc}
\hline Case & Resolution $\left(2^{L}\right)$ & $d_{0} / \lambda$ & We & $\rho_{r}$ & $\mu_{r}$ & Sc & $\operatorname{Re}_{\lambda}$ \\
\hline 1 & $\mathrm{~L} 10 \equiv 2^{10}, \mathrm{~L} 11 \equiv 2^{11}$ & 1.72 & 1.3 & 850 & 25 & $(1,2,10,20,50,100)$ & 38 \\
2 & $\mathrm{~L} 10 \equiv 2^{10}, \mathrm{~L} 11 \equiv 2^{11}$ & 2.30 & 1.3 & 850 & 25 & $(1,2,10,20,50,100)$ & 55 \\
3 & $\mathrm{~L} 10 \equiv 2^{10}, \mathrm{~L} 11 \equiv 2^{11}, \mathrm{~L} 12 \equiv 2^{12}$ & 2.82 & 1.3 & 850 & 25 & $(1,2,10,20,50,100)$ & 77 \\
\hline \hline
\end{tabular}

Table 2: Simulation parameters (with adaptive mesh refinement) of the turbulence simulation of mass transfer. Three Reynolds numbers are used, with two effective resolutions, and a range of Schmidt numbers. The Weber number, density and viscosity ratio are kept constant. 

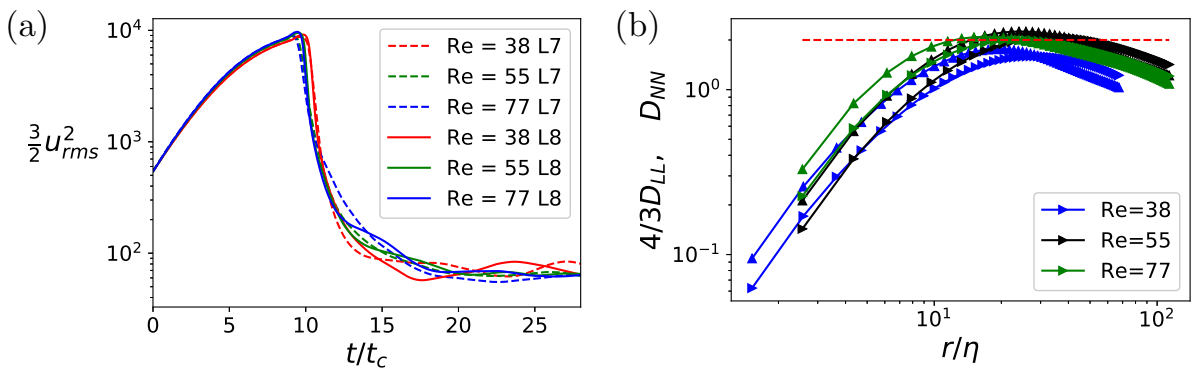

Figure 3: Properties of the homogeneous and isotropic turbulent flow. (a) Turbulent kinetic energy as a function of time. After a short transient, a statistically stationary state is reached. The bubble is inserted once the statistically stationary state is reached. (b) Second-order structure function, $D_{L L}$ and $D_{N N}$ in the longitudinal and transverse directions respectively, compensated by the homogeneous and isotropic turbulence scaling $(r \epsilon)^{-2 / 3}$ and $D_{L L}=3 / 4 D_{N N}$. Turbulence theory $4 / 3 D_{L L}(r)(r \epsilon)^{2 / 3}$ is superimposed in red dashed line. $\eta$ is the Kolmogorov length scale. The bubble has a size comparable to the Taylor turbulence scale, within the inertial range.

\subsection{Precursor simulation for isotropic turbulence}

The turbulent flow is generated by adding a linear volumetric forcing term $f=A \mathbf{u}(\mathbf{x}, t)$ in Eq. 3.1. This approach has been introduced by Rosales \& Meneveau (2005) and yields similar turbulence properties as using a forcing in spectral space and leads to a wellcharacterized homogeneous and isotropic turbulent flow. Such an approach has been applied to study bubble rising in turbulence by Loisy et al. (2017), and more recently we used this approach within the Basilisk solver to study bubble deformation in turbulence (Perrard et al. 2021; Rivière et al. 2021). We consider a 3D periodic box of size $L$, for a precursor simulation to achieve isotropic turbulence. We use adaptive mesh refinement on the velocity field, and the maximum level of refinement can be used to compare the resolution with that of a fixed grid. The turbulent flow is generated for increasing resolutions with the maximum level of refinement going from 6 to 8, corresponding to an equivalent of $64^{3}$ to $256^{3}$ grid points. The resolution will be increased once we insert the bubble. The turbulence state is characterized by the kinetic energy density $K$, turbulence dissipation rate $\epsilon$ and Taylor microscale Reynolds number $\operatorname{Re}_{\lambda}$, which are given by (Pope 2001)

$$
K=\frac{1}{V} \int_{V} \frac{1}{2} \rho_{l}\left|u^{\prime}(\mathbf{x}, t)\right|^{2} d V, \quad \epsilon=\frac{1}{V} \int_{V} \nu_{l}\left(\frac{\partial u_{i}}{\partial x_{j}} \frac{\partial u_{i}}{\partial x_{j}}\right) d V, \quad \operatorname{Re}_{\lambda}=\frac{2 K}{3 \nu_{l}} \sqrt{\frac{15 \nu_{l}}{\epsilon}}
$$

and are computed over time to characterize the turbulent flow. The root mean square of the velocity is $u_{\mathrm{rms}}=\sqrt{2 K / 3 \rho_{l}}$, and the eddy turn over time at the scale of the bubble of diameter $d_{0}$ is given by $t_{c}=d_{0}^{2 / 3} \epsilon^{-1 / 3}$ (Pope 2001; Perrard et al. 2021).

Figure $3(\mathrm{a})$ shows the evolution of the turbulent kinetic energy with time for increasing Reynolds numbers. It shows that at $t / t_{c} \approx 25$ the flow has reached a statistically stationary state. We show that the state is grid-independent when using an adaptive mesh refinement of $\mathrm{L} 7 \equiv 2^{7}$ and $\mathrm{L} 8 \equiv 2^{8}$ for the three cases. The turbulence Reynolds number and the turbulence dissipation rate have similar time evolutions. The range of turbulent Taylor Reynolds numbers is $\operatorname{Re}_{\lambda} \approx 38$ to 77 which is a typical value for current two-phase simulations of turbulent flow (Loisy et al. 2017; Elghobashi 2019b). We characterize the turbulent stationary state using the second-order structure functions 
in the longitudinal $D_{L L}(r)$ and the transverse direction $D_{N N}(r)$, given by $D_{L L}(r)=$ $\frac{1}{3} \sum_{i}\left\langle\left(u_{i}(\mathbf{r}, t)-u_{i}\left(\mathbf{r}+d \hat{\mathbf{r}}_{i}, t\right)\right)^{2}\right\rangle$, and $D_{N N}(r)=\frac{1}{6} \sum_{i \neq j}\left\langle\left(u_{i}(\mathbf{r}, t)-u_{i}\left(\mathbf{r}+d \hat{\mathbf{r}}_{j}, t\right)\right)^{2}\right\rangle$, where $\hat{\mathbf{r}}_{i}$ is the unit vector along the $\mathrm{i}^{\text {th }}$ direction. Figure $3(\mathrm{~b})$ shows that the scaled structure functions plateau at $C=2$ (Pope 2001) in the inertial range. The relation $D_{L L}=3 / 4 D_{N N}$ is verified and the inertial range is relatively limited due to the relatively coarse resolution and limited turbulence Reynolds number. The bubble is inserted once the turbulent stationary state is reached, and is of a size within the inertial range, the turbulence at this scale being reasonable, as described in (Perrard et al. 2021; Rivière et al. 2021).

\subsection{Bubble insertion}

The bubble is inserted at the center of the box after reaching isotropic turbulence, i.e. for $t_{0}>25 t_{c}$ (See Fig. 3(a)). The bubble is of diameter $d_{0}$, viscosity $\mu_{b}$, and density $\rho_{b}$ surrounded by a liquid of viscosity $\mu_{l}$ and density $\rho_{l}$. The solubility of dilute gas is $\alpha_{j}=$ 0.3 which is transferred across the interface. The Weber number, We $\equiv \rho_{l} u_{\mathrm{rms}}^{2} d_{0} / \gamma=1.3$ is below the critical number for bubble breakup (Perrard et al. 2021; Rivière et al. 2021), so that all results are for bubbles that can deform but do not break. Theoretical discussion and experimental data in Theofanous et al. (1976) provide the approximation that the transfer rate is a weak function of surface tension within 3 orders of magnitude $(0.001 \lesssim$ $W e \lesssim 1)$ and depends mainly on the bulk turbulence properties. Diffusion rates for six different gases are calculated corresponding to Schmidt numbers ranging from 1 to 100 . The bubble size with respect to the Taylor micro-scale length $\left(\lambda=\sqrt{15 \nu u_{\mathrm{rms}}^{2} / \epsilon}\right)$ is in the range $1.72-2.82$, and the box size is $L=7.5 d_{0}$. The turbulence properties and simulation parameters are given in Table 2. Th higher resolutions are used to properly resolve the diffusion of mass and the bubble deformation dynamics, with effective resolution using an adaptive mesh refinement of $\mathrm{L} 10 \equiv 2^{10}, \mathrm{~L} 11 \equiv 2^{11}$ and $\mathrm{L} 12 \equiv 2^{12}$. As will be discussed in detail later, higher Schmidt numbers lead to thinner diffusive boundary layers which require smaller grid sizes. The resolution of smallest momentum and mass length scales are discussed in appendix A.

\subsection{Mass transfer from the bubble to the surrounding turbulent flow}

As the bubble moves with the flow, vortices of outer liquid come in contact with the interface, as seen in Figures 4(a)-4(b). Molecular diffusion of gas around the bubble interacts with the eddies of the flow and unsteady boundary layers are formed. The corresponding concentration field for $\mathrm{Sc}=1$ and $\mathrm{Sc}=10$ are shown in figures $4(\mathrm{c})-4(\mathrm{~d})$ and 4(e)-4(f). It can be observed that the concentration fields have followed the flow field due to advection. The gas with high diffusivity $(\mathrm{Sc}=1)$ has thicker boundary layers (Figures 4(c) and (d)) compared to the low diffusivity gas (Sc $=10$, Figures 4(e) and (f)) subjected to the exact same flow. Figure 5 shows a $3 \mathrm{D}$ rendering of the same times of the simulations, displaying the 3D concentration field around the bubble, as the bubble moves in the flow, with the lower $\mathrm{Sc}=1$ in figures 5(a)-5(b), and higher $\mathrm{Sc}=10$ in figures $5(\mathrm{c})-5(\mathrm{~d})$. Again, higher Schmidt numbers lead to thinner boundary layers around the bubble for the same flow field.

We next compute the total transfer rates $k_{L}$ of gases corresponding to increasing Schmidt numbers, and show their evolution with time in Fig. 6 for increasing numerical resolution, $d_{0} / \Delta x=136$ points per bubble diameter $(\mathrm{a}, \mathrm{b})$ and $d_{0} / \Delta x=273$ (points per bubble diameter (c,d) and increasing turbulence Reynolds number $\operatorname{Re}_{\lambda}=38$ (a,c) and $\operatorname{Re}_{\lambda}=77(\mathrm{~b}, \mathrm{~d})$. The time is shifted to the bubble insertion time $t_{0}$ and normalized with the eddy turn over time $t_{c}$. After a short transient, the transfer rates reach a steady 
(a)

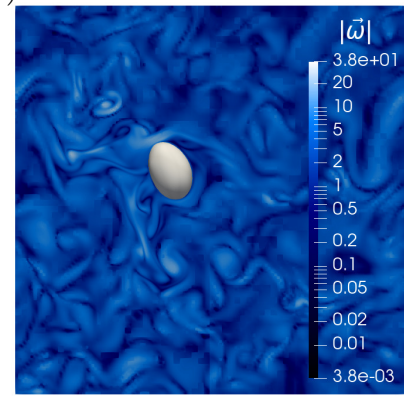

(c)

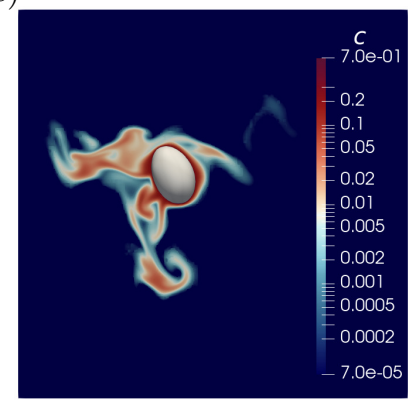

(e)

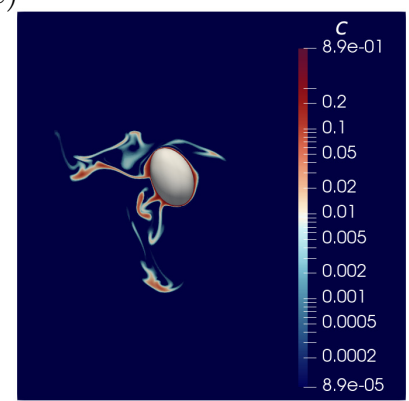

(f) (b)

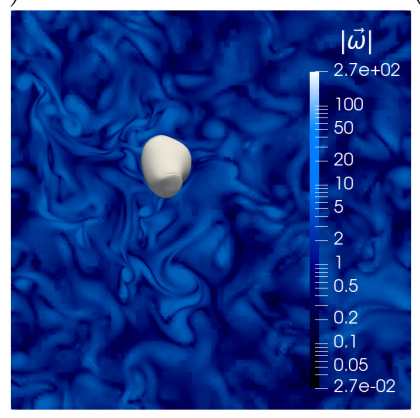

(d)

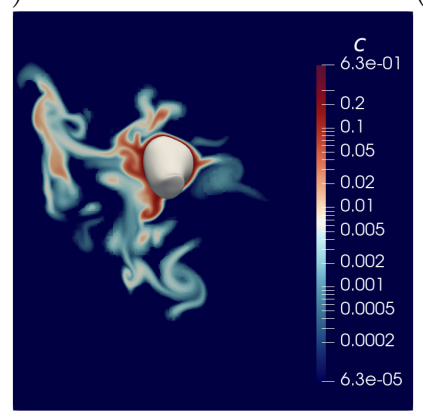

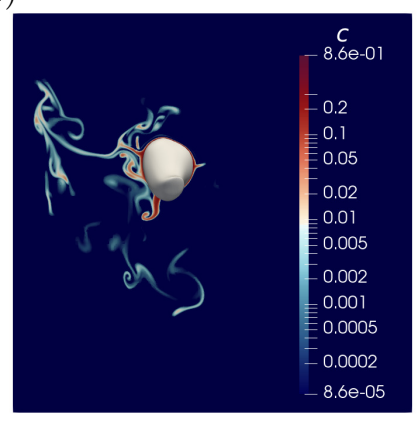

Figure 4: Mass diffusion from the bubble in turbulence at two different times, $\left(t-t_{0}\right) / t_{c}=$ 1.5 (top row) and 2 (bottom row), showing a $2 \mathrm{D}$ planar cut of the magnitude of the vorticity field (a)-(b) and concentration field for $\operatorname{Re}_{\lambda}=77, \mathrm{Sc}=1\left(P e^{(t)}=205\right)(\mathrm{c})$-(d) and $\operatorname{Re}_{\lambda}=77, \mathrm{Sc}=10\left(P e^{(t)}=2050\right)(\mathrm{e})$-(f). The wake in the vorticity field presents similarities with the structure of the concentration field. Higher Schmidt numbers lead to a thinner boundary layer around the bubble and a thinner wake structure.

state for $\left(t-t_{0}\right) / t_{c}>0.25$. The transfer rates in steady state are compared to the predicted rates given by Eq. (2.3), which are shown as dashed lines. At lower resolution and Reynolds number (panel a, $d_{0} / \Delta x=136$ and $\operatorname{Re}_{\lambda}=38$ ), all cases exhibit good agreement between computed and predicted transfer rates, and results are unchanged when the resolution is increased (see panel c, $d_{0} / \Delta x=273$ and $\operatorname{Re}_{\lambda}=38$ ). The decrease in rates over time for lower-Schmidt-number cases in figure $6(\mathrm{a})$ and $6(\mathrm{~b})$ is due to a considerable decrease in concentration of the transferred dilute gas. When increasing the Reynolds number, higher resolution is required for the highest Schmidt numbers, as visible in $\mathrm{b}$ and $\mathrm{c}$. To summarize, the DNS results are in close agreement with the predicted rates for $50<\widetilde{\mathrm{Pe}}<10^{4}$, with $\widetilde{\mathrm{Pe}}=u_{\mathrm{rms}} d_{0} / \mathscr{D}_{l}$ the turbulent Péclet number.

For higher turbulent Péclet numbers $\left(1.02 \times 10^{4}, 2.05 \times 10^{4}\right)$, we observe an overprediction of transfer rates. These results for the cases of $\widetilde{\mathrm{Pe}}>10^{4}$ (yellow and black curves in figure 6(b) and 6(d)) can be understood by considering the resolution of boundary layers. The thickness of the hydrodynamic boundary layer, $\delta_{\nu}$ around a spherical bubble is of order $\mathcal{O}\left(\mathrm{Re}^{-1 / 2}\right)$ (Moore 1963). The concentration boundary layer thickness $\delta_{k} \propto$ $\delta_{\nu} \mathrm{Sc}^{-1 / 2}$ (Levich 1962; Bothe \& Fleckenstein 2013), scales with the bubble diameter $d_{0}$, and is given by, $\delta_{k} / d_{0} \approx \mathrm{Pe}^{-1 / 2}$. The numerical framework used in the present work uses adaptive mesh refinement with respect to the norm of the second-derivative of velocity and concentration (van Hooft et al. 2018), with an error threshold of 0.2 times the average of fields over the entire domain. The average of concentration fields are very small as 
(a)

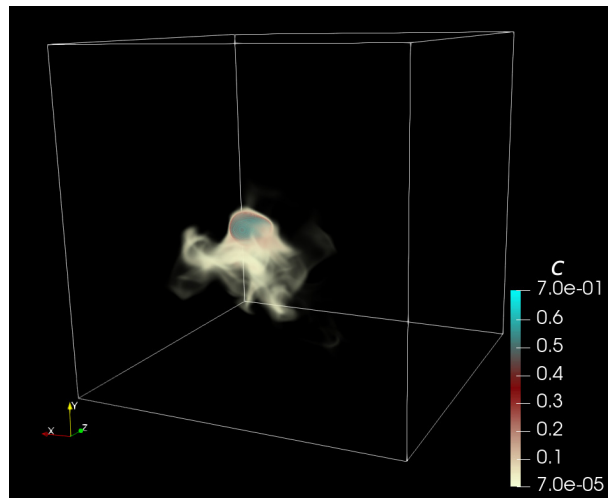

(b)

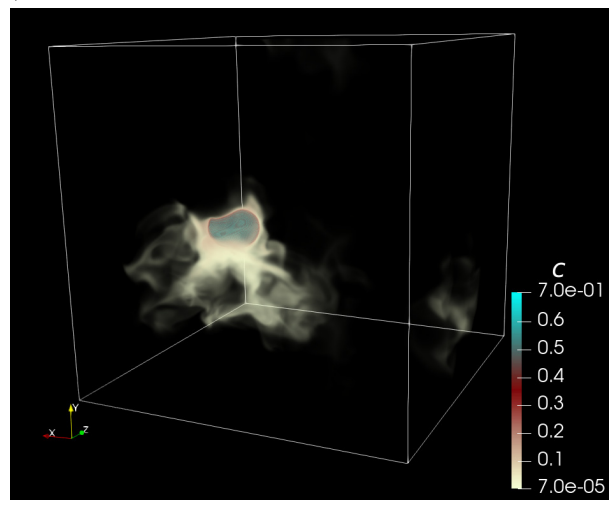

(c)

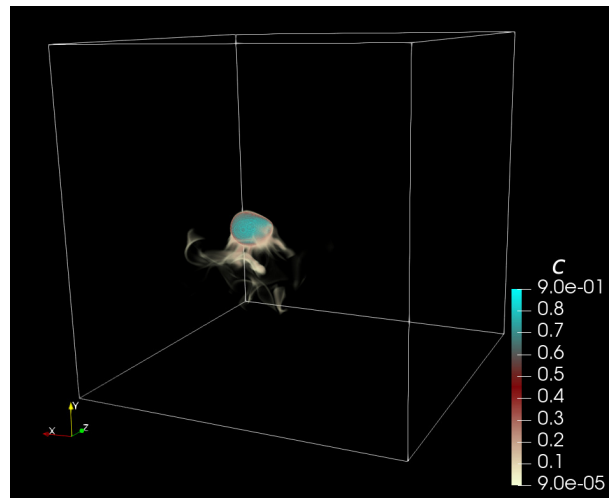

(d)

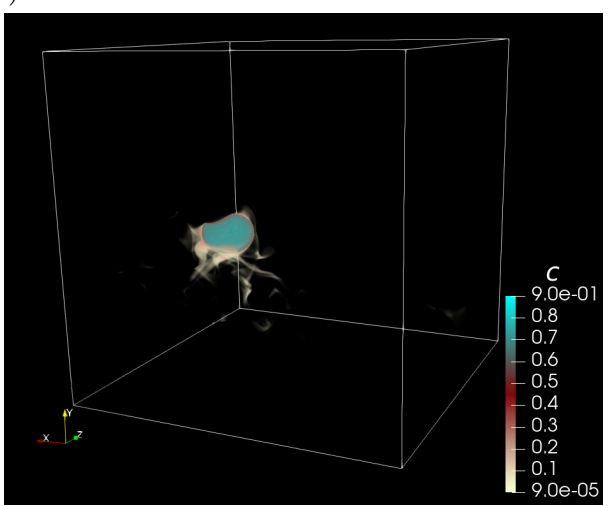

Figure 5: 3D rendering of the concentration field for $\left(t-t_{0}\right) / t_{c}=1.5$ (top row) and 2 (bottom row). (a)-(b) Concentration field for $\operatorname{Re}_{\lambda}=77, \mathrm{Sc}=1\left(P e^{(t)}=205\right)$ and (c)-(d) Concentration field for $\operatorname{Re}_{\lambda}=77, \mathrm{Sc}=10\left(\mathrm{Pe}^{(t)}=2050\right)$. Thinner boundary layers are observed at higher Schmidt numbers.

most of the domain has trace amounts gas concentration. This leads to a resolution of the boundary layers ranging from 40 grid points $\left(\widetilde{\mathrm{Pe}} \approx 50, \delta_{\nu} / d_{0} \approx 0.13, \delta_{k} / d_{0} \approx 0.13\right)$ to 3 grid points $\left(\widetilde{\mathrm{Pe}} \approx 2 \times 10^{4}, \delta_{\nu} / d_{0} \approx 0.07, \delta_{k} / d_{0} \approx 0.007\right)$. When the resolution is increased, we are able to resolve thinner boundary layers, hence higher Péclet numbers $\left(1.02 \times 10^{4}, 2.05 \times 10^{4}\right)$, as shown in figure $6(\mathrm{~b})$ and $6(\mathrm{~d})$ where the rates for $\widetilde{\mathrm{Pe}}>10^{4}$ are converging to the predicted value as the resolution increases (L11 in solid lines and L12 in dotted lines).

Finally, Figure 7 shows the Sherwood numbers obtained from the bubble mass transfer in homogeneous and isotropic turbulence simulations for a wide range of turbulent Péclet numbers $\left(50<\widetilde{\mathrm{Pe}} \leq 2 \times 10^{4}\right)$ for three different resolutions (simulations conditions are summarized in table 2). Very good agreement is observed between Eq. (2.3), up to $\widetilde{\mathrm{Pe}} \sim 10^{4}$. As such, Figure 7 validates Eq. (2.3), which predicts the transfer rates from the bubble in homogeneous and isotropic turbulent flow. We note that for $\mathrm{Pe} \approx 2 \times 10^{4}$, an effective resolution L12 brings the simulated transfer rate closer to our theoretical prediction, thanks to increased resolution in the turbulent-diffusive boundary layer next to the bubble interface. We recall that Figure 7 shows the scaling for the transfer rate $k_{L}$, 

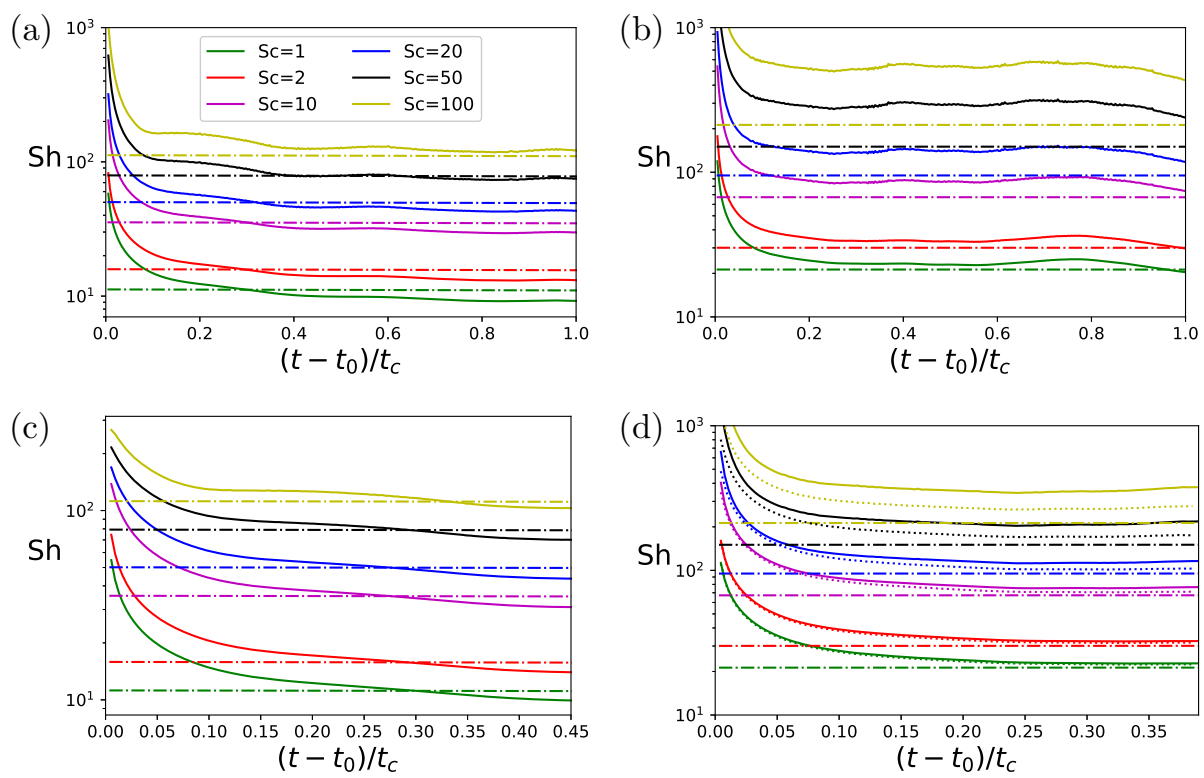

Figure 6: Non-dimensional mass transfer rates Sh as a function of time, as the bubble is exposed to the turbulent flow. (a,b) Lower resolution $d_{0} / \Delta x=136$ (level 10), for $\operatorname{Re}_{\lambda}=$ 38 (a) and $\operatorname{Re}_{\lambda}=77$ (b). (c,d) Higher resolution (solid line for level $11 d_{0} / \Delta x=273$, dotted line for level $12 d_{0} / \Delta x=546$ ), for $\operatorname{Re}_{\lambda}=38$ (c) and $\operatorname{Re}_{\lambda}=77$ (d). Dashed lines represent the theoretical prediction, Eq. 2.3 for the different Schmidt numbers. At steady state, very good agreement between simulations and theory is achieved for $\widetilde{\mathrm{Pe}}=u_{\mathrm{rms}} d_{0} / \mathscr{D}_{l}=\operatorname{Sc}\left(u_{\mathrm{rms}} d_{0} / \mathscr{D}_{l}\right) \leq 10^{4}$, which corresponds to a diffusive boundary layer $\delta_{k}$ resolved with more than $\sim 4$ grid points.

as the non-dimensional Sh number, which reads $S h \propto \operatorname{Re}^{0.5} \mathrm{Sc}^{0.5}=\sqrt{\widetilde{\mathrm{Pe}}}$, and considering the definition of the Sh number, this corresponds to $k_{L} \propto \mathrm{Sc}^{-0.5} \sqrt{\frac{u_{\mathrm{rms}} \nu_{l}}{d_{0}}}$.

We note that Dodd et al. (2021) presents simulations of a droplet evaporating in turbulence and their evaporation rates at early times before significant volume change has occurred are in agreement with our model, as shown in figure 7.

The computed Sherwood numbers show convergence to the predicted value as the grid resolution increases. For the thinner boundary layers, corresponding to $\operatorname{Re}_{\lambda}=77$ and high Schmidt number at $d_{0} / \Delta x=273$ resolution, the total number of cells in the domain reached 27.5 million and used $2.16 \times 10^{4} \mathrm{CPU}$ hours for 0.5 eddy turnover time when the transfer rates approach a steady state. A corresponding constant-resolution direct numerical simulation would have required $\left(2^{11}\right)^{3} \approx 8.6$ billion cells, which illustrates the gain in efficiency brought by adaptive mesh refinement. It highlights the potential of adaptive mesh refinement methods for complex two-phase flows at high Peclet number, complementary to sub-grid scale modeling approaches.

We note that in $\S 2$, we have proposed the large-scale velocity fluctuations as the controlling velocity scale. As already mentioned, another choice could have been the velocity fluctuations at the scale of the bubble. Such a choice would not have changed the results obtained in figure 7 , as these velocities are similar at the Reynolds number at the Taylor-scale we consider.

Finally let us comment that at high Reynolds numbers, in the case of a flat interface, 


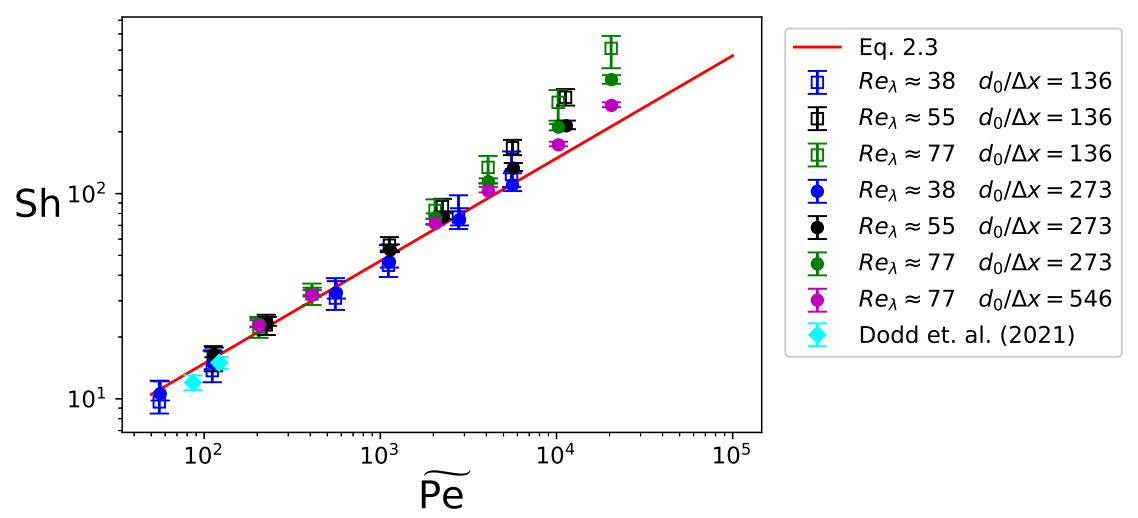

Figure 7: The transfer rates (for $\left(t-t_{0}\right) / t_{c} \geq 0.2$ ) with respect to Péclet number. Symbols are for different $\mathrm{Re}_{\lambda}$ and resolution. For $\widetilde{\mathrm{Pe}} \leq 10^{4}$, the numerical results are grid converged (first order) between L10 and L11, and agree very well with the theoretical prediction, Eq. (2.3) $\mathrm{Sh}=2(3)^{1 / 4} \sqrt{\widetilde{\mathrm{Pe}} / \pi}$. For higher Péclet numbers $\left(1.02 \times 10^{4}, 2.05 \times 10^{4}\right)$, higher resolutions may converge to the prediction as visible when using L12 simulations. The transfer rates from Dodd et al. (2021) corresponds to the transfer rates from simulations of an evaporating drop at early times and agree with our data and model.

the scaling of the gas transfer is shifted from $\mathrm{Re}^{1 / 2}$ to $\mathrm{Re}^{3 / 4}$ due to the role of small eddies in enhancing the gas exchange (Theofanous et al. 1976; Herlina \& Wissink 2016). Whether such a regime could be observed in the case of bubble mass exchange at high Peclet or Reynolds number remains to be investigated.

\section{Concluding remarks}

We propose a simple theoretical formula for mass transfer rate of dilute gas from bubbles for a dilute component within a turbulent flow, extending the classic formulation from Higbie (1935) to a homogeneous and isotropic turbulent flow, by considering that the renewal time-scale is controlled by the turbulence fluctuations at large scale and the bubble size:

$$
\mathrm{Sh}=\frac{2(3)^{1 / 4}}{\sqrt{\pi}} \sqrt{\widetilde{\mathrm{Pe}}}
$$

We developed and presented a versatile numerical framework to perform direct numerical simulations of multiphase flows, and tested the theoretical prediction against numerical simulations. The agreement between theory and numerical results is very good, as long as the diffusive boundary layer thickness is correctly resolved. The numerical framework can now be used for more complex physical configurations such as bubbles under a breaking wave and bubble swarms.

The proposed theoretical scaling for dilute mass transfer is remarkable as it could be leveraged for multi-scale systems, such as bubbles entrained by breaking waves in the upper ocean, or bubbles evolving in turbulent swarms, and avoid solving for the very high Schmidt number necessary in practical problems (for example the Schmidt number of $\mathrm{CO}_{2}$ at 20 degrees is $\sim 660$ ). Finally, our formulation could also be used to improve recent bubble-mediated mass transfer models which have, up to now, used the bubble transfer rate in a quiescent flow (Liang et al. 2011; Deike \& Melville 2018). 
As part of this work, a video has been published in the gallery of fluid motion (Farsoiya et al. 2020c).

\section{Acknowledgments}

This work was supported by the NSF CAREER award 1844932, the Catalysis Initiative at Princeton and the Cooperative Institute for Modeling the Earth System (CIMES) between Princeton and NOAA-GFDL. We would like to acknowledge highperformance computing support from Tiger, Princeton research computing and Cheyenne (doi:10.5065/D6RX99HX) provided by NCARs Computational and Information Systems Laboratory, sponsored by the National Science Foundation. Declaration of Interests. The authors report no conflict of interest.

\section{Appendix A}

The accepted resolution criteria for DNS in the literature (Overholt \& Pope 1996; Pope 2001; Schumacher et al. 2005; Dodd et al. 2021) is typically $k_{\max } \eta>1.5$ and $k_{\max } \eta_{B}>1.5$, where $k_{\max }, \eta$ and $\eta_{B}$ are the maximum resolved wavenumber $k_{\max }=$ $\pi N / L$, the Kolmogorov and Batchelor scale respectively. The Kolmogorov length scale $\eta=\left(\nu_{l}^{3} / \epsilon\right)^{1 / 4}$ defines the length scale at which viscous dissipation becomes dominant while the Batchelor scale is defined as $\eta_{B}=\eta / \sqrt{S c}$. Figure 8 shows these characteristic length scales as functions of the turbulent Peclet number for all our simulations. In all of the cases the Kolmogorov length scale is well resolved, with $k_{\max } \eta>8$, in agreement with the fact that convergence is already achieved in lower resolutions as shown in figure 3a. For the highest Péclet number, the Batchelor length scale is resolved up to $k_{\max } \eta_{B} \approx 3.2$ (refinement $d_{0} / \Delta x=546$ ). The boundary layer thickness $\delta_{\nu}>2.5 \eta$ and $\delta_{k}>2.5 \eta_{B}$.
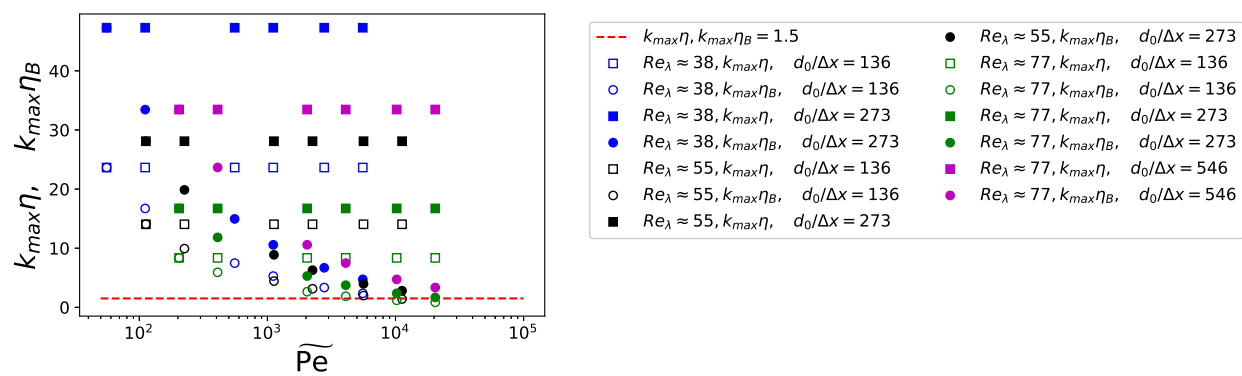

Figure 8: Numerical resolution of Kolmogorov $(\eta)$ and Batchelor $\left(\eta_{B}\right)$ length scales as functions of Peclet number, normalized by the maximum resolved wavenumber $k_{\max }=$ $\pi N / L$.

\section{REFERENCES}

Berny, Alexis, Deike, Luc, Séon, Thomas \& Popinet, Stéphane 2020 Role of all jet drops in mass transfer from bursting bubbles. Physical Review Fluids 5 (3), 033605.

Bird, R, Stewart, W \& Lightfoot, E 2002 Transport phenomena-second edition.

Bothe, Dieter \& Fleckenstein, Stefan 2013 A volume-of-fluid-based method for mass transfer processes at fluid particles. Chemical Engineering Science 101, 283-302.

Bothe, D, Koebe, M, Wielage, K, Prüss, J \& Warnecke, H-J 2004 Direct numerical 
simulation of mass transfer between rising gas bubbles and water. In Bubbly Flows, pp. 159-174. Springer.

Boussinesq, J 1905 Calcul du poivoir refroidissant des courants fluide. J. Math. Pures Appl. 60, 285-332.

Brackbill, Jeremiah U, Kothe, Douglas B \& Zemach, Charles 1992 A continuum method for modeling surface tension. Journal of computational physics 100 (2), 335-354.

Cano-Lozano, José Carlos, Martinez-Bazan, Carlos, Magnaudet, Jacques \& TCHOufag, JoËL 2016 Paths and wakes of deformable nearly spheroidal rising bubbles close to the transition to path instability. Physical Review Fluids 1 (5), 053604.

Claassen, Claire My, Islam, Shafiul, Peters, EAJf, Deen, Niels G, Kuipers, JAM \& Baltussen, Maike W 2020 An improved subgrid scale model for front-tracking based simulations of mass transfer from bubbles. AIChE Journal 66 (4), e16889.

Clift, Roland, Grace, John R \& Weber, Martin E 2005 Bubbles, drops, and particles. Courier Corporation.

Colombet, Damien, Legendre, Dominique, Risso, Frédéric, Cockx, Arnaud \& Guiraud, PASCAL 2015 Dynamics and mass transfer of rising bubbles in a homogenous swarm at large gas volume fraction. Journal of Fluid Mechanics 763, 254-285.

Darmana, Dadan, Deen, Niels G \& Kuipers, JAM 2006 Detailed 3d modeling of mass transfer processes in two-phase flows with dynamic interfaces. Chemical Engineering 83 Technology: Industrial Chemistry-Plant Equipment-Process Engineering-Biotechnology 29 (9), 1027-1033.

Davidson, Malcolm R \& Rudman, Murray 2002 Volume-of-fluid calculation of heat or mass transfer across deforming interfaces in two-fluid flow. Numerical Heat Transfer: Part B: Fundamentals 41 (3-4), 291-308.

Deike, Luc \& Melville, W. Kendall 2018 Gas transfer by breaking waves. Geophysical Research Letters 45 (19), 10,482-10,492.

Deike, Luc, Melville, W. Kendall \& Popinet, Stéphane 2016 Air entrainment and bubble statistics in breaking waves. Journal of Fluid Mechanics 801, 91-129.

Deising, D, Bothe, D \& Marschall, H 2018 Direct numerical simulation of mass transfer in bubbly flows. Computers \& Fluids 172, 524-537.

Deising, Daniel, Marschall, Holger \& Bothe, Dieter 2016 A unified single-field model framework for volume-of-fluid simulations of interfacial species transfer applied to bubbly flows. Chemical Engineering Science 139, 173-195.

Dodd, Michael S \& Ferrante, Antonino 2016 On the interaction of taylor length scale size droplets and isotropic turbulence. Journal of Fluid Mechanics 806, 356.

Dodd, Michael S, Mohaddes, Danyal, Ferrante, Antonino \& Ihme, Matthias 2021 Analysis of droplet evaporation in isotropic turbulence through droplet-resolved dns. International Journal of Heat and Mass Transfer 172, 121157.

Elghobashi, SAID 2019a Direct numerical simulation of turbulent flows laden with droplets or bubbles. Annual Review of Fluid Mechanics 51, 217-244.

Elghobashi, SAID $2019 b$ Direct numerical simulation of turbulent flows laden with droplets or bubbles. Annual Review of Fluid Mechanics 51 (1), 217-244.

Farsoiya, Palas, Popinet, Stephane \& Deike, Luc 2020a Diffusion from static bubble. http://basilisk.fr/sandbox/farsoiya/static_bubble.c, [Online; accessed 02-Nov2020].

Farsoiya, Palas, Popinet, Stephane \& Deike, Luc 2020b Diffusion from rising bubble. http://basilisk.fr/sandbox/farsoiya/axi_rising_bubble.c, [Online; accessed 02Nov-2020].

Farsoiya, Palas, Popinet, Stephane \& Deike, Luc 2020c Gas transfer from bubble in isotropic turbulence. DOI:https://doi.org/10.1103/APS.DFD.2020.GFM.V0012, [Online; accessed 19-Nov-2020].

Farsoiya, Palas Kumar, Mayya, YS \& Dasgupta, Ratul 2017 Axisymmetric viscous interfacial oscillations-theory and simulations. Journal of Fluid Mechanics 826, 797-818.

Farsoiya, Palas Kumar, Roy, Anubhab \& Dasgupta, Ratul 2020 Azimuthal capillary waves on a hollow filament-the discrete and the continuous spectrum. Journal of Fluid Mechanics $\mathbf{8 8 3}$.

Fleckenstein, Stefan \& Bothe, Dieter 2015 A volume-of-fluid-based numerical method 
for multi-component mass transfer with local volume changes. Journal of Computational Physics 301, 35-58.

Fortescue, GE \& PeArson, JRA 1967 On gas absorption into a turbulent liquid. Chemical Engineering Science 22 (9), 1163-1176.

Gumulya, M, Utikar, RP, Pareek, VK, Evans, GM \& Joshi, JB 2020 Dynamics of bubbles rising in pseudo-2d bubble column: Effect of confinement and inertia. Chemical Engineering Journal 405, 126615.

HAROun, Y, LEGENDRE, D \& RAYNAL, L 2010 Volume of fluid method for interfacial reactive mass transfer: application to stable liquid film. Chemical Engineering Science 65 (10), 2896-2909.

HeRLinA, H \& Wissink, JG 2016 Isotropic-turbulence-induced mass transfer across a severely contaminated water surface. Journal of Fluid Mechanics 797, 665-682.

HeRlina, H \& Wissink, JG 2019 Simulation of air-water interfacial mass transfer driven by high-intensity isotropic turbulence. Journal of Fluid Mechanics 860, 419-440.

Higbie, RALPH 1935 The rate of absorption of a pure gas into a still liquid during short periods of exposure. Trans. AIChE 31, 365-389.

van Hooft, J Antoon, Popinet, Stéphane, van Heerwahrden, Chiel C, van der Linden, Steven JA, De Roode, Stephan R \& van de Wiel, Bas JH 2018 Towards adaptive grids for atmospheric boundary-layer simulations. Boundary-layer meteorology 167 (3), 421-443.

Jia, Hongwei, Xiao, Xin \& Kang, Yanming 2019 Investigation of a free rising bubble with mass transfer by an arbitrary lagrangian-eulerian method. International Journal of Heat and Mass Transfer 137, 545-557.

Karn, Ashish, Monson, Garrett M, Ellis, Christopher R, Hong, Jiarong, Arndt, Roger EA \& GulLiver, John S 2015 Mass transfer studies across ventilated hydrofoils: A step towards hydroturbine aeration. International Journal of Heat and Mass Transfer 87, 512-520.

KAtul, Gabriel \& Liu, HePing 2017 Multiple mechanisms generate a universal scaling with dissipation for the air-water gas transfer velocity. Geophysical Research Letters 44 (4), $1892-1898$.

KeELING, RALPH F 1993 On the role of large bubbles in air-sea gas exchange and supersaturation in the ocean. Journal of Marine Research 51 (2), 237-271.

Levich, V G 1962 Physicochemical hydrodynamics .

Liang, Jun-Hong, McWilliams, James C, Sullivan, Peter P \& Baschek, Burkard 2011 Modeling bubbles and dissolved gases in the ocean. Journal of Geophysical Research: Oceans 116 (C3).

Loisy, Aurore, Naso, Aurore \& Spelt, Peter DM 2017 Buoyancy-driven bubbly flows: ordered and free rise at small and intermediate volume fraction. Journal of Fluid Mechanics 816, 94-141.

López-Herrera, JM, Ganan-Calvo, AM, Popinet, S \& Herrada, MA 2015 Electrokinetic effects in the breakup of electrified jets: A volume-of-fluid numerical study. International Journal of Multiphase Flow 71, 14-22.

Maes, Julien \& Soulaine, Cyprien 2020 A unified single-field volume-of-fluid-based formulation for multi-component interfacial transfer with local volume changes. Journal of Computational Physics 402, 109024.

Magnaudet, Jacques \& Calmet, Isabelle 2006 Turbulent mass transfer through a flat shear-free surface. Journal of Fluid Mechanics 553, 155.

Marić, Tomislav, Kothe, Douglas B \& Bothe, Dieter 2020 Unstructured un-split geometrical volume-of-fluid methods-a review. Journal of Computational Physics 420, 109695.

Marschall, Holger, Hinterberger, Korbinian, Schüler, Christian, Habla, Florian \& HinRiCHSEN, OlAF 2012 Numerical simulation of species transfer across fluid interfaces in free-surface flows using openfoam. Chemical engineering science 78, 111-127.

Mathai, Varghese, Lohse, Detlef \& Sun, Chao 2020 Bubble and buoyant particle laden turbulent flows. Annu. Rev. Condens. Matter Phys 11.

Maxworthy, T, Gnann, C, Kürten, M \& Durst, F 1996 Experiments on the rise of air bubbles in clean viscous liquids. Journal of fluid mechanics 321, 421-441. 
Moore, DW 1963 The boundary layer on a spherical gas bubble. Journal of Fluid Mechanics 16 (2), 161-176.

Moore, DW 1965 The velocity of rise of distorted gas bubbles in a liquid of small viscosity. Journal of Fluid Mechanics 23 (4), 749-766.

Mostert, W \& Deike, L 2020 Inertial energy dissipation in shallow-water breaking waves. Journal of Fluid Mechanics $\mathbf{8 9 0 .}$.

Overholt, MR \& Pope, SB 1996 Direct numerical simulation of a passive scalar with imposed mean gradient in isotropic turbulence. Physics of Fluids 8 (11), 3128-3148.

Perrard, Stphane, Rivire, Alinor, Mostert, Wouter \& Deike, Luc 2021 Bubble deformation by a turbulent flow, arXiv: 2011.10548.

Pope, Stephen B 2001 Turbulent flows.

Popinet, StÉPhane 2009 An accurate adaptive solver for surface-tension-driven interfacial flows. Journal of Computational Physics 228 (16), 5838-5866.

Popinet, StÉPhane 2015 A quadtree-adaptive multigrid solver for the serre-green-naghdi equations. Journal of Computational Physics 302, 336-358.

Popinet, StÉphane 2018 Numerical models of surface tension. Annual Review of Fluid Mechanics 50, 49-75.

Popinet, StÉPhane \& COllaborators 2013-2020 Basilisk. http://basilisk.fr.

Rachin, Azeddine, Legendre, Dominique, Climent, Eric \& Charton, Sophie 2020 Numerical study of conjugate mass transfer from a spherical droplet at moderate reynolds number. International Journal of Heat and Mass Transfer 157, 119958.

Reichl, Brandon G \& Deike, Luc 2020 Contribution of sea-state dependent bubbles to air-sea carbon dioxide fluxes. Geophysical Research Letters p. e2020GL087267.

RIsso, FrÉDÉRIC 2018 Agitation, mixing, and transfers induced by bubbles. Annual Review of Fluid Mechanics 50, 25-48.

Rivière, Aliénor, Mostert, Wouter, Perrard, Stéphane \& Deike, Luc 2021 Sub-hinze scale bubble production in turbulent bubble break-up. Journal of Fluid Mechanics 917.

Roghair, I 2012 Direct numerical simulations of hydrodynamics and mass transfer in dense bubbly flows .

Rosales, Carlos \& Meneveau, Charles 2005 Linear forcing in numerical simulations of isotropic turbulence: Physical space implementations and convergence properties. Physics of fluids 17 (9), 095106.

Ruth, D., Mostert, W., Perrard, S. \& Deike, L. 2019 Bubble pinch-off in turbulence. PNAS .

SANDER, Rolf 2015 Compilation of henry's law constants (version 4.0) for water as solvent. Atmospheric Chemistry and Physics 15 (8), 4399-4981.

SAto, T, Jung, R-T \& ABE, S 2000 Direct simulation of droplet flow with mass transfer at interface. J. Fluids Eng. 122 (3), 510-516.

Scapin, Nicolò, Costa, Pedro \& Brandt, Luca 2020 A volume-of-fluid method for interfaceresolved simulations of phase-changing two-fluid flows. Journal of Computational Physics 407, 109251.

Scardovelli, Ruben \& Zaleski, StéPhane 1999 Direct numerical simulation of free-surface and interfacial flow. Annual review of fluid mechanics 31 (1), 567-603.

Schumacher, J, Sreenivasan, KR \& Yeung, PK 2005 Very fine structures in scalar mixing. Journal of Fluid Mechanics 531, 113-122.

StAndART, G 1964 The mass, momentum and energy equations for heterogeneous flow systems. Chemical Engineering Science 19 (3), 227-236.

Tanguy, Sébastien, Sagan, Michaël, Lalanne, Benjamin, Couderc, Frédéric \& Colin, CATHERINE 2014 Benchmarks and numerical methods for the simulation of boiling flows. Journal of Computational Physics 264, 1-22.

TAQIEDDIN, AmIR 2018 Modeling of bubbles hydrodynamics and mass transfer in electrochemical gas-evolving systems. PhD thesis, Northeastern University.

Theofanous, TG, Houze, RN \& Brumfield, LK 1976 Turbulent mass transfer at free, gasliquid interfaces, with applications to open-channel, bubble and jet flows. International Journal of Heat and Mass Transfer 19 (6), 613-624.

Treybal, Robert E 1980 Mass transfer operations. New York 466. 
Tryggvason, Grétar, Scardovelli, Ruben \& Zaleski, Stéphane 2011 Direct numerical simulations of gas-liquid multiphase flows. Cambridge University Press.

Weiner, Andre \& Bothe, Dieter 2017 Advanced subgrid-scale modeling for convectiondominated species transport at fluid interfaces with application to mass transfer from rising bubbles. Journal of Computational Physics 347, 261-289.

Whitman, Walter G 1923 The two-film theory of gas absorption. Chem. Metall. Eng. 29, $146-148$.

Wolfram Research, Inc. 2020 Mathematica, Version 12.1. https://www.wolfram.com/ mathematica, champaign, IL, 2020.

Woolf, DAvid K \& Thorpe, SA 1991 Bubbles and the air-sea exchange of gases in nearsaturation conditions. Journal of Marine Research 49 (3), 435-466.

Yang, Lei, Peters, EAJF, Fries, L, Harshe, YM, Kuipers, JAM \& Baltussen, Maike W 2020 Direct numerical simulation of mass transfer and mixing in complex two-phase systems using a coupled volume of fluid and immersed boundary method. Chemical engineering science: $X \mathbf{5}, 100059$. 